Relations industrielles

Industrial Relations

\title{
Personnel Management and Industrial Relations. By Dale
} YoDER. 4th Edition, Englewood Cliffs, N.J.: Prentice-Hall, Inc., 1956. $941 \mathrm{pp}$.

\section{Roger Chartier}

Volume 11, numéro 3, juin 1956

URI : https://id.erudit.org/iderudit/1022638ar

DOI : https://doi.org/10.7202/1022638ar

Aller au sommaire du numéro

\section{Éditeur(s)}

Département des relations industrielles de l’Université Laval

\section{ISSN}

0034-379X (imprimé)

1703-8138 (numérique)

Découvrir la revue

Citer ce compte rendu

Chartier, R. (1956). Compte rendu de [Personnel Management and Industrial Relations. By Dale Yoder. 4th Edition, Englewood Cliffs, N.J.: Prentice-Hall, Inc., 1956. 941 pp.] Relations industrielles / Industrial Relations, 11(3), 223-224.

https://doi.org/10.7202/1022638ar

Tous droits réservés @ Département des relations industrielles de l’Université Laval, 1956
Ce document est protégé par la loi sur le droit d'auteur. L’utilisation des services d'Érudit (y compris la reproduction) est assujettie à sa politique d'utilisation que vous pouvez consulter en ligne.

https://apropos.erudit.org/fr/usagers/politique-dutilisation/ 
Money and Motivation: An Analysis of Incentives in Industry. By William $\mathrm{F}$. Whyte et al. New York: Harper \& Bros., 1955, XII, 268 pp. $\$ 4$.

Les plans de rémunération du travail in dustriel selon la production individuelle - dits "incentive plans》, «plans boni », etc. - datent de plusieurs années déjà, et sont fort répandus dans l'industrie nord-américaine et européenne surtout. Ils se rattachent à un vaste effort d's organisation scientifique du travail » et de la rémunération ouvrière. Ingénieurs industriels et économistes, comme il se doit, leur ont toujours porté un intérêt grand et consacré des milliers de volumes et d'articles. Money and Motivution apporte un élément neuf, présente un point de vue à la fois original et utile: il est l'oeuvre de sociologues et de psychologues.

Le volume se présente bien; le style est simple, la langue, claire. Whyte vise décidément un vaste public. Les spécialistes y trouveront cependant leur compte eux aussi, tout en retrouvant sous la plume de lauteur principal des énoncés de faits et des donnés théoriques déjà publiés ailleurs. Je pense à l'article de Collins, Dalton et Roy sur la restriction de la production. (Applied Anthropology, V., 3), suivi d'autres articles dans la même veine par ces mêmes auteurs, puis par Whyte lui-même, Sayles et Strauss - auteurs de The Local Union - et enfin par Frank Miller, Alex Vavelas et Friedrich Fuerstenberg.

Money and Motivation, cependant, n'est pas un ramassis d'articles vieillis alignés en vrac. Whyte a puisé généreusement aux sources précitées, mais ii les a intégrés avec art, leur ajoutant de très nombreux éléments factuels et théoriques nouveaux.

Nous n'avons pas ici un volume de recettes sur les stimulants économiques dans l'industrie à l'usage d'un employeur pressé par des échéances immédiates et peu avide de discussions sur les fondements psycho-sociologiques des divers plans de rémunération selon la production. Whyte - avec l'aide de ses collaborateurs - s'intéresse d'abord à la réalité totale de l'organisation industrielle, dont la comoréhension est essentielle à l'évaluation des «incentive plans ». Cette réalité-là, il va sans dire, dépasse de beaucoup les considérations purement économiques. débouchant sur les motivations d'une masse d'hommes en continuelle interaction.
La première partie du volume décrit la façon dont le travailleur et son groupe exercent un contrôle sur les plans de stimulants à la production - leur « éducation » au contrôle, l'organisation sociale de ce contrôle et des négociations plus ou moins articulées qui le préparent, les avantages et inconvénients sociaux et psychologiques de pareils plans, etc.

La deuxième partie analyse l'influence des plans de stimulants, non plus sur les relations entre individus à l'intérieur d'un même groupe de travail, mais sur les relations entre groupes différents de travail à l'intérieur d'un même établissement, v.g. entre divers départements, entre tel département et la direction à tel ou tel niveau. On y trouve également un cas d'espèce démontrant les bouleversements dans l'organisation spontanée d'un département produits par l'introduction d'un plan de stimulants à la production, ainsi qu'une étude du complexe de significations du gain chez les travailleurs.

La troisième partie s'attache à l'analyse de ce système social plus vaste qu'est l'usine elle-même, en tant qu'il est influenoé par le «plan boni ».

La quatrième partie, théorique à fond, reprend plusieurs concepts structures, symboles, relations intergroupes et individus-groupes, sentiments, activités, interaction - précédemment énoncés, les raffine et les situe dans un cadre de référence logique, utilisant à la fois ce cadre et les faits décrits pour bátir et vérifier des hypothèses nombreuses sur l'organisation humaine dans le cadre industriel.

Un volume qu'il faut lire à tout prix.

Personnel Management and Industrial Relations. By Dale Yoder. 4th Edition. Englewood Cliffs, N.J.: Prentice-Hall, Inc., 1956. $941 \mathrm{pp}$.

Voici la quatrième édition, complètement revue et corrigée, du livre à succès du professeur Yoder. La première, intitulée Personnel and Labor Relations, date déià de 1938. La troisième a huit ans. En tout, plus de 281,000 exemplaires ont été vendus. Sur ce point, aucun doute: le livre a réussi. Il nous est cependant difficile de souscrire pleinement à - lassertion de lauteur que ces 281,000 exemplaires ont beaucoup contribué à l'amélioration des relations patronales-ouvrières. 
Le professeur Yoder a décidément le don de la vulgarisation. C'est un simplificateur-né. Sous sa plume, tout se dilue, tout devient simple, tout s'explique derechef; d'où sa réussite dans le genre manuel introductif. En fait, nous aurions été plus vite rassuré si un autre que Yoder, avec autorité, avait fait la revision!

A la décharge de l'auteur, il importe de déclarer que cette nouvelle édition contient de nombreuses améliorations sur la précédente. En huit ans, tellement de choses surviennent dans le domaine des relations industrielles! L'auteur en a sérieusement fait son profit, et ce manuel gagne en poids, en agencement, en volume aussi.

A l'usage de l'étudiant qui veut pénétrer sans trop de chocs dans le domaine de la direction du personnel et des relations industrielles, qui ne se pose pas trop de questions et qui ne désire pas trop de réponses.

Psychology of Personnel in Business and Industry. By Roger M. Bellows. 2nd Edition. New York: Prentice-Hall, Inc., 1954. 467 pp. $\$ 5.50$.

Ce volume s'adresse d'abord aux administrateurs d'entreprises industrielles et commerciales, et particulièrement aux directeurs du personnel. Dans cette édition, l'auteur a réussi à faire plus concis que dans la première (1949), ce qui est à la fois douloureux et méritoire. Le volume est mieux divisé, et les références sont à date.

Sous l'angle de la psychologie appliquée, l'auteur analyse tour à tour les différentes méthodes et techniques de création ou d'amélioration de la satisfaction ouvrière, et d'organisation « efficace du personnel. On trouve dans ce livre des commentaires utiles sur le « counseling », l'évaluation du «moral » des travailleurs, l'analyse des tâches, le recrutement, l'entrevue, la sélection, les tests, la formation - technique ou autre - de la main-d'oeuvre, la notation (merit rating) du personnel, les stimulants à la production, et ainsi de suite.

En dépit d'une certaine dispersion qui donne dans la superficialité, le volume a du mérite et vaut d'être lu.
Union Strike Votes: Current Practice and Proposed Controls. By Herbert S. Parnes. Princeton: Industrial Relations Section, Princeton University, 1956. $141 \mathrm{pp}$.

Notre législation est censée favoriser la négociation collective; d'autre part, le droit de grève est une condition (normalement) essentielle du régime de la convention collective. On concoit donc limportance qu'il y a à étudier le procédé par lequel la grève s'institue.

L'opinion publique accepte assez facilement, bien souvent, le fait non vérifié du manque de démocratie syndicale, du défaut fréquent à instituer le vote de grève, du besoin pour le gouvernement de venir «protéger» les membres individuels écrasés par les structures syndicales.

Le professeur Parnes, s'appuyant sur les pratiques de vote de grève de 135 syndicats locaux, particulièrement dans le New Jersey, a des vues beaucoup plus optimistes.

La plupart des syndicats étudiés exigent un vote de grève majoritaire au scrutin secret. La plupart des chefs syndicaux - et des gars du rang voient dans la prise du vote un geste utile et prudent. Et ainsi de suite dans le même sens.

A lire, pour détruire certains préjugés faciles et voir la réalité telle qu'elle est, du moins dans les cas étudiés.

The Sociology of Industrial Relations: An Introduction to Industrial Sociology. By John B. Knox. New York: Random House, 1955. 348 pp.

Le livre du professeur Knox est le dernier en date des volumes généraux portant sur la sociologie industrielle. Je ne pense pas aux travaux des Lynds, de lEcole de Mayo, de Warner \& Associates, mais plutôt à l'Industrial Sociologu de Miller \& Form - qui fait terriblement ramasse-tout - au volume de Wilbert Moore sur les Industrial Relations and the Social Order - un classique qui a su systématiser dans un cadre logique les données sociologiques acquises dans le domaine industriel - et enfin à l'oeuvre, assez faible et plutôt mal nommée, de Theodore Caplowf sur The Sociology of Work (U. of Minnesota, 1954).

Le professeur Knox a des ambitions modestes: il ne s'adresse pas au sociolo- 